\title{
OPEN Contrast enhanced X-ray computed tomography imaging of amyloid plaques in Alzheimer disease rat model on lab based micro CT system
}

\author{
Michaela Kavkova $^{1 凶}$, Tomas Zikmund ${ }^{1}$, Annu Kala ${ }^{2}$, Jakub Salplachta ${ }^{1}$, \\ Stephanie L. Proskauer Pena ${ }^{2}$, Josef Kaiser ${ }^{1} \&$ Karel Jezek ${ }^{2}$
}

Amyloid plaques are small $(\sim 50 \mu \mathrm{m})$, highly-dense aggregates of amyloid beta $(A \beta)$ protein in brain tissue, supposed to play a key role in pathogenesis of Alzheimer's disease (AD). Plaques' in vivo detection, spatial distribution and quantitative characterization could be an essential marker in diagnostics and evaluation of AD progress. However, current imaging methods in clinics possess substantial limits in sensitivity towards $A \beta$ plaques to play a considerable role in $A D$ screening. Contrast enhanced X-ray micro computed tomography (micro CT) is an emerging highly sensitive imaging technique capable of high resolution visualization of rodent brain. In this study we show the absorption based contrast enhanced X-ray micro CT imaging is viable method for detection and 3D analysis of $A \beta$ plaques in transgenic rodent models of Alzheimer's disease. Using iodine contrasted brain tissue isolated from the Tg-F344-AD rat model we show the micro CT imaging is capable of precise imaging of $A \beta$ plaques, making possible to further analyze various aspects of their 3D spatial distribution and other properties.

Alzheimer's disease $(\mathrm{AD})$ is the most common cause of dementia worldwide, extensively interfering with personal, social, and health care levels of human society. AD progression is characterized by a gradual deterioration of cognitive abilities, starting with often unnoticed declarative memory impairment, followed by problems with spatial orientation, and ending with an inability to cope with daily life routine. The rare early onset form can affect individuals in their $40 \mathrm{~s}$ or $50 \mathrm{~s}$, but the AD risk rapidly increases after the age of 65 . While the etiology of $\mathrm{AD}$ remains still unclear, there are several hypothesized mechanisms reflecting molecular changes observed in brain tissue of $\mathrm{AD}$ patients, mainly the deposits of amyloid beta and hyperphosphorylated tau protein ${ }^{1-3}$. The classical "amyloid cascade hypothesis"4 states that the amyloid beta $(\mathrm{A} \beta)$ plaques are the causative agent of Alzheimer's pathology. The $A \beta$ plaques are small $(\sim 50 \mu \mathrm{m})$, dense objects composed of clumped fibrils of amyloid $\beta(A \beta)$, a peptidic product of the amyloid precursor protein. Their formation induces a local inflammation resulting in progressive cellular loss and related cognitive inability. It has been therefore assumed that the reduction of $\mathrm{A} \beta$ deposits might help to control the $\mathrm{AD}$ course. Numerous approaches have been designed to reduce the $A \beta$ content in the brain tissue $e^{5}$. In order to test the effectiveness of such an AD therapy, there is a substantial need of a non-invasive, precise, and reproducible imaging method to analyze the location and size of the $\mathrm{A} \beta$ plaques in the brain.

In the large majority of studies on mouse and rat models, the amyloid plaques were quantified histologically in sliced and stained brain tissue. While the histology and immunohistochemistry still remain important routines for characterizing the plaque properties, imaging techniques represent a powerful tool for a precise 3D analysis of plaque distribution.

So far all the attempts to image the amyloid plaques in rodent brain by Computed Tomography (CT) have been performed on mouse brain (mus musculus). The experiments were performed with synchrotron X-ray sources using variations of phase contrast imaging to visualize the $A \beta$ plaques $^{6-11}$. Overall, in all published

${ }^{1}$ Central European Institute of Technology, Brno University of Technology, Brno, Czech Republic. ${ }^{2}$ Faculty of Medicine in Pilsen, Charles University, Pilsen, Czech Republic. ${ }^{\square}$ email: michaela.kavkova@ceitec.vutbr.cz; karel.jezek@Ifp.cuni.cz 
papers on the topic of $A \beta$ plaques detection, the authors were able to identify the $A \beta$ plaques in the mouse brain using phase contrast imaging, some even performing quantitative analyses ${ }^{11}$. However, imaging at synchrotron facilities is often limited by the narrow field of view resulting in a need of several scans for the imaging of one brain. Also, the access to synchrotron facilities is very limited and burdened by the high price of the CT scans.

Absorption based contrast enhanced X-ray micro CT imaging utilizing a lab based industrial micro CT devices is an emerging imaging technique which uses the staining solutions with high proton number elements to employ the contrast in soft tissues of biological samples which are in their native state invisible to X-rays ${ }^{12,13}$. Contrast enhanced micro CT imaging has been previously proved to be a promising method for a precise 3D analysis of wide variety of samples such as the developing cartilage of nasal capsule of mice ${ }^{14-17}$, development of palate ${ }^{18,19}$, complex tooth shape in reptiles ${ }^{20}$, research of congenital heart and kidneys defects ${ }^{21}$, formation of mammalian neck muscles ${ }^{22}$ and even a noninvasive observation of a human embryo ${ }^{23}$. One of the advantages of micro CT imaging is the possibility to translate a micro CT generated 3D model into a $3 \mathrm{D}$ pdf format, which enables an easier communication of obtained data ${ }^{24}$.

The main advantage of absorption based contrast enhanced X-ray micro CT imaging is the possibility to obtain precise $3 \mathrm{D}$ information about inner structures of the entire brain without sectioning (which might induce artifacts related to brain deformation or missing tissue due to the sectioning). The micro CT imaging of rodent brain has been previously used for the visualization of vascular system of mouse brain filled with radio-opaque silicone rubber Microfil ${ }^{25-31}$, as a tool for localization of cerebral ischemia ${ }^{32,33}$ to test the efficiency of different micro CT contrasting agents ${ }^{24-38}$, and even for the analysis of specific structures of the brain ${ }^{39}$.

However, it has not been previously determined whether the absorption based contrast enhanced X-ray micro CT imaging is capable of detecting and quantifying amyloid plaques in the brains of rodents. In this study, we optimized the mouse brain staining protocol ${ }^{40}$ for the staining of a much larger rat brain. The lab based industrial micro CT device GE Phoenix v|tome|x L 240 was utilized to scan the contrast enhanced brains of a recent rat $\mathrm{AD}$ model to determine whether amyloid plaques could be detected by contrast enhanced micro CT imaging. Further, the exploration of the possibilities of $\mathrm{A} \beta$ plaque analyses provided by obtained high resolution micro $\mathrm{CT}$ data was pursued. This experimental setup promises a faster, more precise and accessible alternative to the synchrotron based micro CT imaging of the A $\beta$ plaque deposits.

\section{Results}

We used brains from three 18 months old female rats (two transgenic TgF-344 AD animals and one wild type control). The micro CT scan slices of iodine stained brains from transgenic and wild type samples are shown in the Fig. 1a (for an animated movie see the Supplementary material). Besides the basic anatomical structures visualized equally across both brains, the transgenic brain expressed a large amount of dark grey spots-most likely suspect $\mathrm{A} \beta$ plaques that were condensed widely in cortical (neocortex, hippocampus) and some subcortical areas.

Beta amyloid is the main component of amyloid plaques. Because of their dense protein content, we expected the applied iodine based contrasting protocol would preferably stain the tissue around the amyloid plaques, resulting in their "negative" highlight in the micro CT data. The golden standard for beta amyloid detection is its immunostaining by binding to a specific antibody tagged by fluorescent probe. Here, we used this approach to validate the identity of lower density loci detected by micro CT in the transgenic samples. To confirm the identity of the suspect plaques, the iodine stained brain samples were washed in ethanol solution after the micro CT scanning, cut into histological sections and finally specifically stained for the amyloid protein. For selected histological slices we identified their respective sections in the micro CT data. The resulting comparison is displayed in Fig. 1b which shows the corresponding slice as a micro CT image (1b) and as a histological section (Fig. 1c, detailed view in Fig. 1d). The placements of the suspect $A \beta$ plaques overlapped with the immunodetected $\mathrm{A} \beta$ plaques positions (arrows in Fig. 1d). This match strongly supports the fact that the introduced micro CT protocol sufficiently detects individual amyloid plaques in ex vivo whole brain.

In the industrial micro CT setups that use the cone beam geometry, the dimension of the sample is one of the main factors that determine the resulting voxel size of the obtained dataset. To acquire a 3D distribution of $\mathrm{A} \beta$ plaques in the best possible details, we decided to image an isolated part of the brain and to scan it again with a smaller voxel size. We focused on the dorsal hippocampus as this structure is severely impaired by the amyloid accumulation and cellular loss in AD. The dissected dorsal part of the hippocampus is showed in Fig. 2a,b. While the whole brain scan delivered a voxel size of $9 \mu \mathrm{m}$ (Fig. 2c), in the case of isolated hippocampus we achieved a voxel dimension of $3 \mu \mathrm{m}$ (Fig. 2d). The comparison of both scans convincingly shows that the readability of the large amyloid plaques' borders and especially the visibility of small plaques was compromised, even though they were distinguishable in the whole brain scan. On the contrary, the dissected sample yielded a considerably higher level of detail, enabling to detect a large amount of plaques of various sizes.

The next step was designed to assess the sensitivity of amyloid plaques detection using micro CT in contrast to the standard immunohistological staining. After the CT measurement, we sliced the isolated hippocampal tissue sample. Despite the dehydrated and iodine stained tissue turned out to be highly fragile while slicing, we were able to select four well preserved tissue sections and immunostained them for the A $\beta$ presence. Then the corresponding micro CT sections were identified and their match was evaluated (Fig. 3a,b). The areas of $\mathrm{A} \beta$ deposit cross sections were marked by different observers. Each of the micro CT/histology image pairs was treated as independent. We obtained the plaque area median value of $597.9 \mu^{2}$ (IQR $862.3 \mu \mathrm{m}^{2}$ ) from CT data, whereas only $28.9 \mu \mathrm{m}^{2}$ (IQR $105.4 \mu \mathrm{m}^{2}$ ) from the histological sections, respectively. The plaque size histograms are depicted in Fig. 3c. This showed a considerable sensitivity difference between both methods, as the immunodetection returned more than one order higher amount of $A \beta$ plaque sections in the lowest size category $\left(0-500 \mu \mathrm{m}^{2}\right)$, accounting for its low median values. Besides the invisibility of smallest deposits on the scans, the comparison of the detailed images in Fig. 3a,b showed that the plaque border was harder to read in the micro CT 

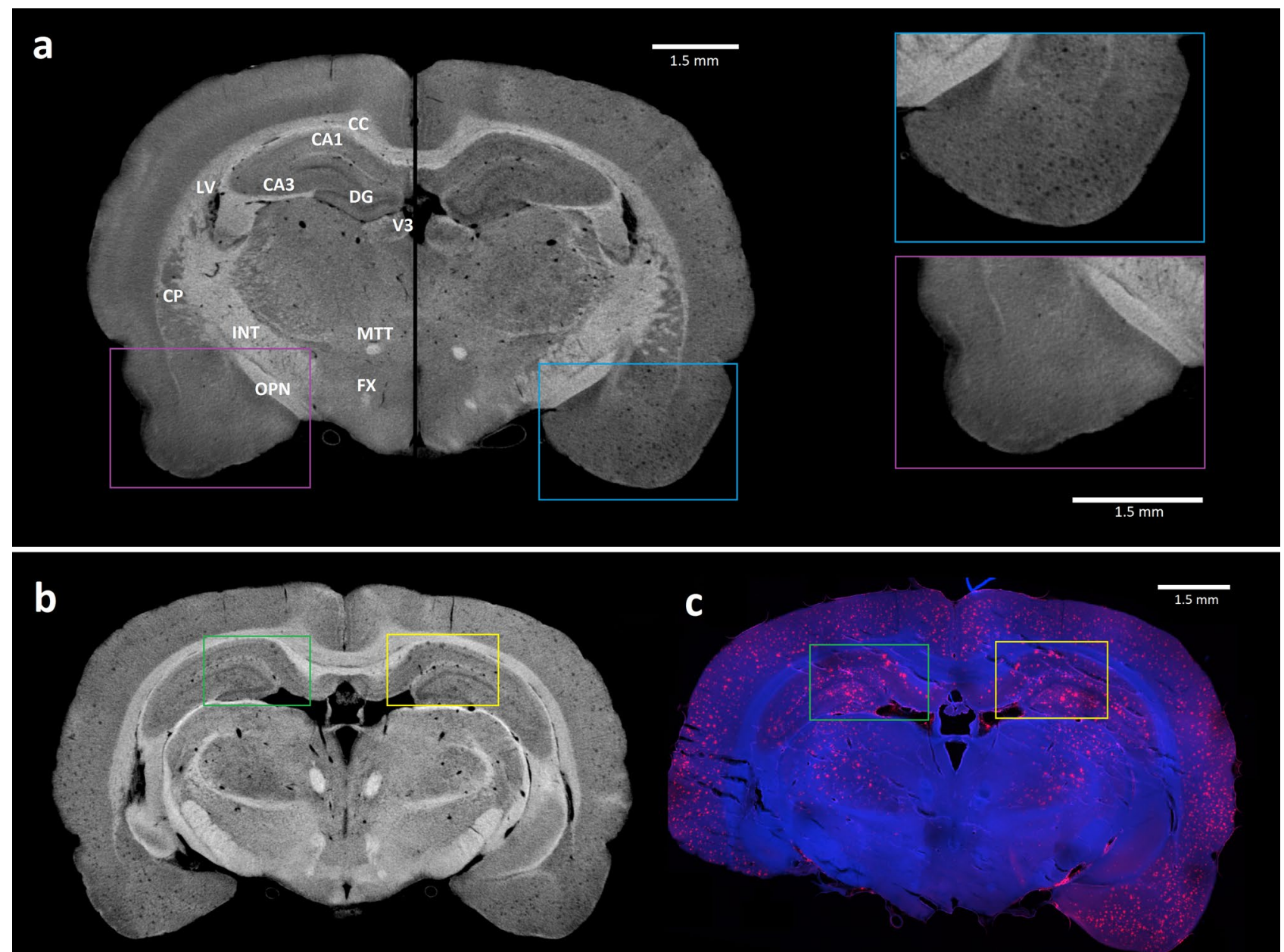

d
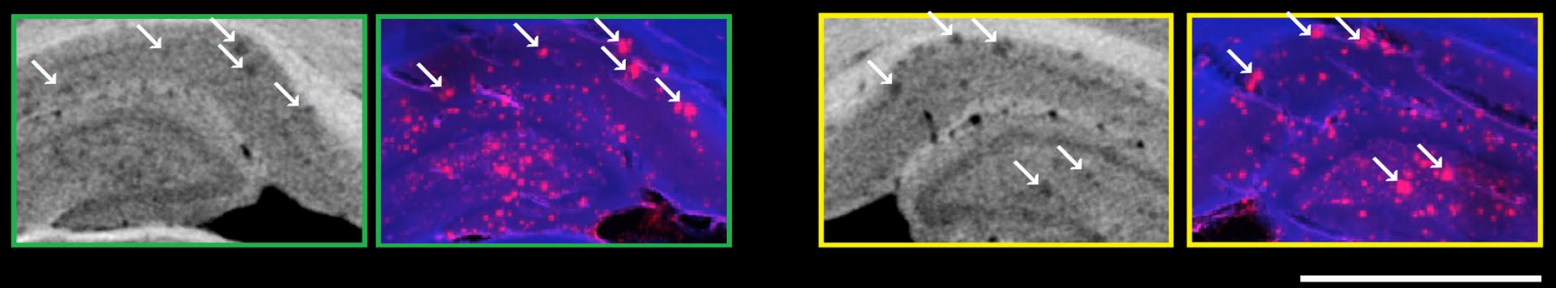

$1.5 \mathrm{~mm}$

Figure 1. Confirmation of plaques identity in micro CT data: (a) Composed picture of the micro CT scan sections from control (left side) and transgenic (right side) rat brain. The transgenic tissue exhibits a large amount of suspect amyloid plaques (right part of the brain). Selected detail image of corresponding areas that are shown in pink and blue frames. Basic anatomical structures visualized in micro CT scan: CA1 and CA3 cornu Ammonis 1 and 3 of hippocampus, $C C$ corpus callosum, $C P$ caudoputamen, $D G$ dentate gyrus, $F X$ fornix, $I N T$ internal capsule, $L V$ lateral ventricle, $M T T$ mamillothalamic tract, $O P N$ olivary pretectal nucleus, V3 third ventricle. Micro CT image (b) and immunohistology section with detected plaques (c) of corresponding coronal brain sections from the transgenic rat no. 60 confirms the presence of plaques in micro CT scan. Selected plaques are indicated by white arrows in enlarged slice windows $(\mathbf{d})^{41}$.

data. This also might have caused an overestimation of the sizes of some of the smallest deposits. Consequently, they might have been marked as larger and so they were scored within the category of $501-1000 \mu \mathrm{m}^{2}$.

The data obtained from the micro CT scan of the dissected hippocampus was chosen for a subsequent $3 \mathrm{D}$ analysis. Since the A $\beta$ plaques had similar contrast values as other tissue structures, they could not be detected with global thresholding methods. Hence, a manual segmentation of the plaques was performed. After defining the region of interest, all segmented plaques were counted and measured. In the dissected hippocampus (total volume $=13.88 \mathrm{~mm}^{3}$ ) we identified in total 1666 individual plaques. The volume of the smallest individual deposit was $895 \mu \mathrm{m}^{3}$, indicating the lower limit for amyloid plaque identification in the present micro CT data. The biggest identified $A \beta$ plaque had a volume of $721,552 \mu \mathrm{m}^{3}$, the dataset of measured volumes was characterized 


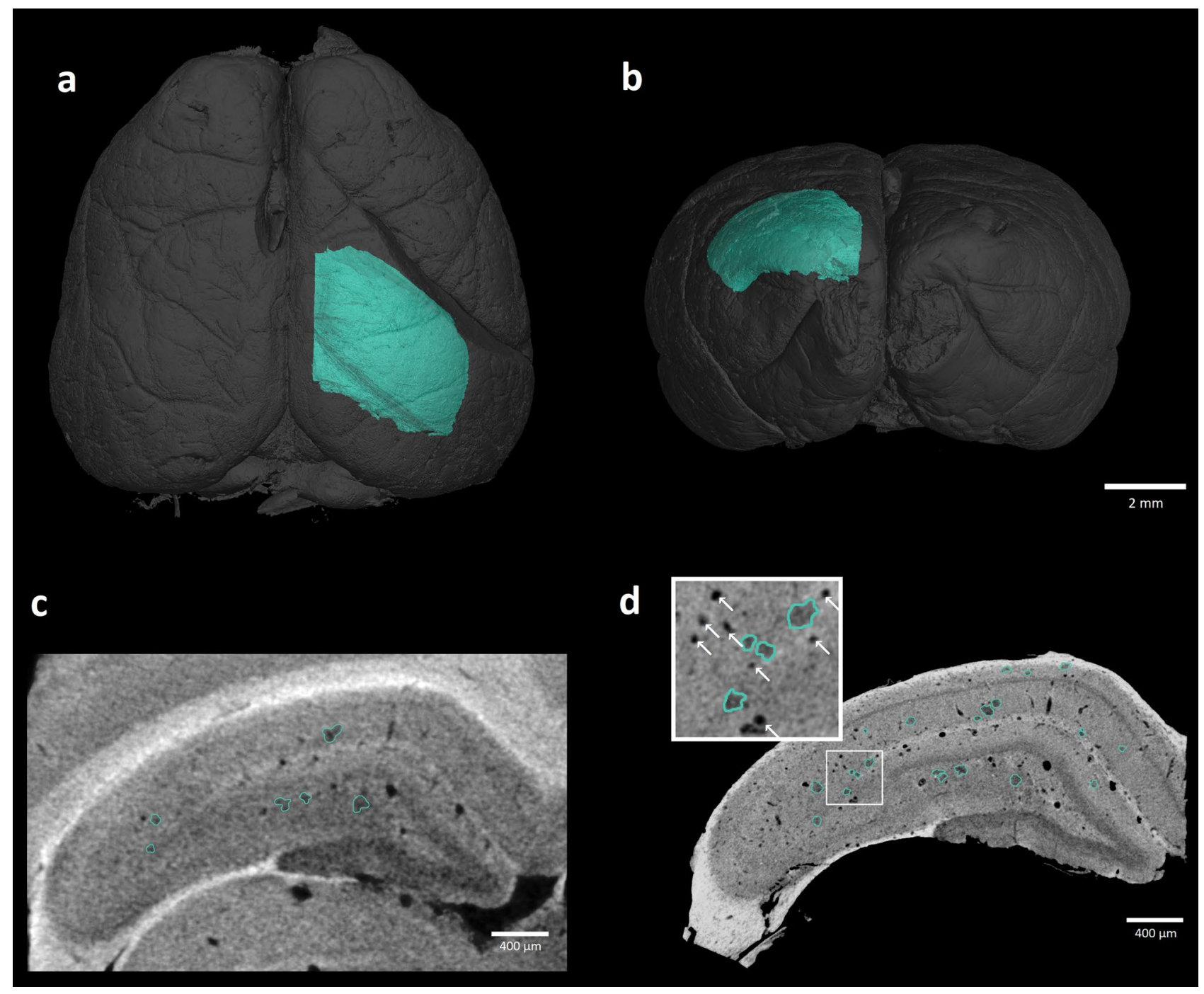

Figure 2. Detailed scan of the dissected dorsal hippocampus: Horizontal (a) and coronal (b) reconstructed view of the brain with dissected right dorsal hippocampus (green) used for the isolated scan. The same hippocampal tissue sample imaged by two different micro CT approaches $(\mathbf{c}, \mathbf{d})$ where the suspect plaques are highlighted in green. Panel (c) shows the section taken from the whole brain scan. Panel (d) depicts the same area after the isolated scanning that achieved smaller voxel size, in the magnified image the blood vessels are labeled by white arrows ${ }^{41,42}$.

by median value $38,423 \mu \mathrm{m}^{3}$ and IQR of $57,512 \mu \mathrm{m}^{3}$. The distribution of the A $\beta$ plaques in $3 \mathrm{D}$ space with their color coded volumes is shown in Fig. $4 \mathrm{a}$. Next, we assessed the shapes' variability of A $\beta$ plaques by measuring their compactness. The volume of the plaque was divided by the volume of the sphere circumscribed to the plaque. The values ranged between $0-1$ where score of one represented a perfect sphere (Fig. $4 \mathrm{~b})$. The identified plaques had median of compactness at 0.396 with interquartile range 0.135 . To alleviate supposed bias that could be caused by an eventual imperfection in defining boundaries in the smaller plaques, we additionally restricted the measurement to subset of plaques with volume larger than its median value ( $50 \%$ of the population). The corresponding compactness had a comparable median of 0.383 and IQR of 0.149 .

A precise $3 \mathrm{D}$ model of the plaque occurrence in the hippocampal sample allowed to quantify their spatial distribution including the relation to other labeled structures. We evaluated the distances of plaques to the nearest blood vessel and the inter-plaque distance (Fig. 5). The reconstructed 3D model returned the median distance between the plaques and the nearest vessel of $64.5 \mu \mathrm{m}$ with IQR $62.3 \mu \mathrm{m}$ (Fig. 5a). We then investigated whether their relation followed a non-random pattern. For each of the 1666 plaques we generated a random coordinate within the dissected part of hippocampus, leaving out the detected blood vessels. We measured the respective distances of generated "plaques" to a nearest vessel with median of $89.1 \mu \mathrm{m}$ and IQR $103 \mu \mathrm{m}$. The comparison between the sets of experimental and randomly generated data returned the plaque-vessel distance significantly shorter in experimental data than in the random sample $(Z=10.95, p<0.001$, Mann Whitney $U$ test), indicating the plaques tended to appear closer to the blood vessels than if their distribution was random. Finally we tested whether the plaques aggregated together irrespectively of the vessels. The same method of generating dataset with random positions was applied, and the distances between the two closest plaques were measured within the 


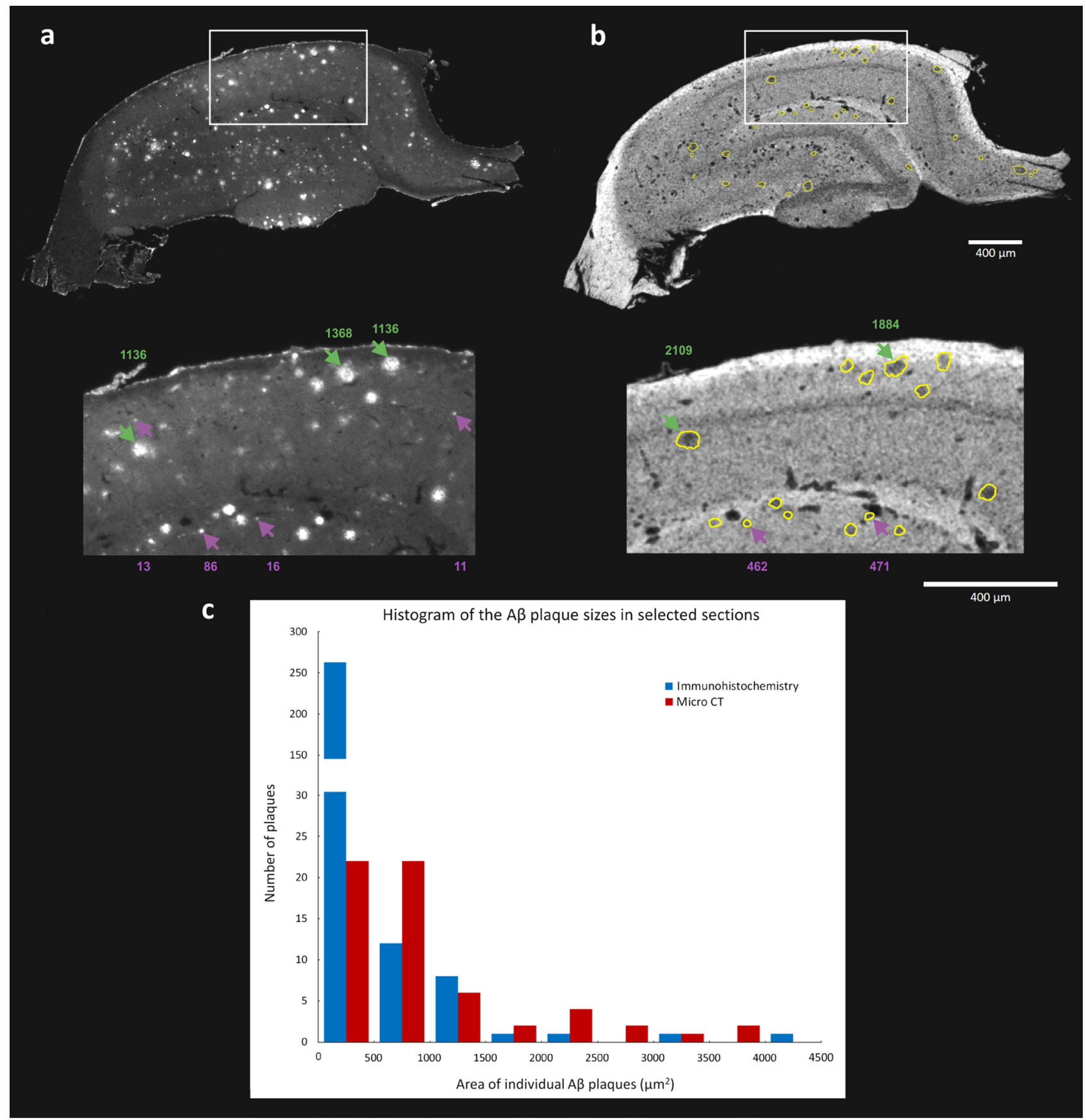

Figure 3. Comparison of the precision in identification of plaques in micro CT data versus the immunohistological detection: Comparison of corresponding sections from the same rat brain showing immunodetected $\mathrm{A} \beta$ plaques (a) and the micro CT data where the yellow line represents the manually selected plaque boundaries (b). The purple arrows indicate examples of the plaque cross sections corresponding to the smallest fraction $\left(0-500 \mu \mathrm{m}^{2}\right)$ while the green arrows mark the larger size cases. The corresponding area values in $\mu \mathrm{m}^{2}$ are reported in proper colors above and under both pictures. The histogram (c) depicts the size interval distribution of detected $A \beta$ plaque sections in the selected slices using immunohistochemistry and micro CT imaging, respectively ${ }^{41-43}$.

experimental and the random positions datasets, respectively (Fig. 5b). The median of distances from the tissue sample data was $101.1 \mu \mathrm{m}$ with $\mathrm{IQR}=46.5 \mu \mathrm{m}$, whereas the randomly generated dataset returned a distribution shift towards larger values (median distance $123.4 \mu \mathrm{m}$ and IQR $62.7 \mu \mathrm{m}$ ). The Mann Whitney U test confirmed a statistically significant difference between the both measurements $(Z=11.97, p<0.001)$. 


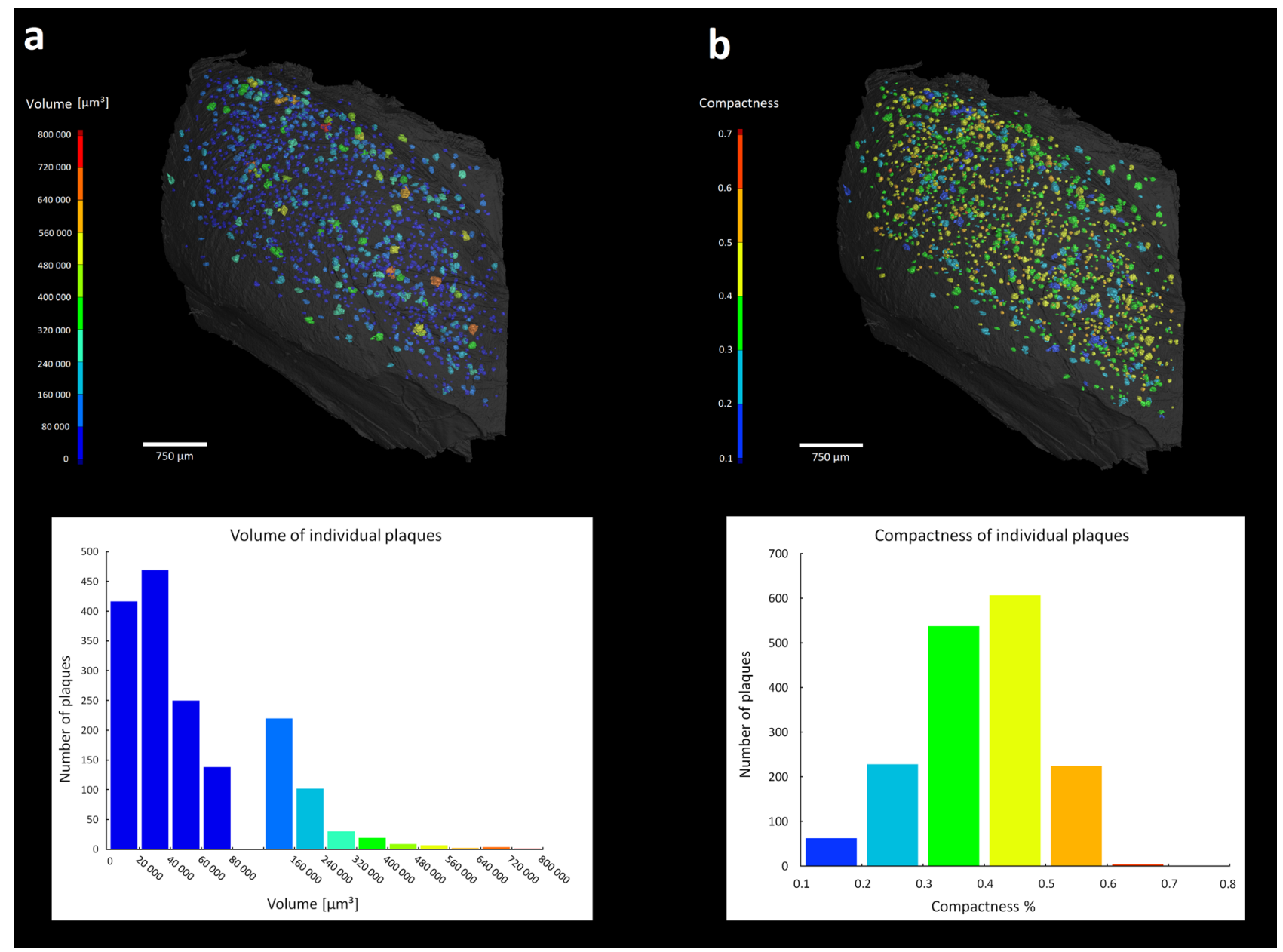

Figure 4. $3 \mathrm{D}$ quantitative analysis of the $\mathrm{A} \beta$ plaques size and shape in hippocampus: Panel (a) shows the plaques $3 \mathrm{D}$ distribution color coded respective to their size, with the corresponding histogram in the same color scale on the right. Panel (b) illustrates the same plaques color scaled according their compactness (red codes for higher similarity to a sphere) with the respective histogram (right) ${ }^{41,42}$.

\section{Discussion}

In this paper, we present the absorption based contrast enhanced X-ray micro CT imaging performed on the industrial lab based CT system as an imaging tool for a $3 \mathrm{D}$ analysis of $\mathrm{A} \beta$ plaques of transgenic rat brains used as a model in Alzheimer disease research.

The detection of amyloid plaques in rodent brain by Computed Tomography $(\mathrm{CT})$ has been attempted so far by using the mouse brain (mus musculus) and the experiments have been performed with synchrotron X-ray sources using variations of phase contrast imaging to visualize the $\mathrm{A} \beta$ plaques in a brain. TOMCAT beamline of the Swiss Light Source, Astolfo et al. ${ }^{6}$ demonstrated that synchrotron-based X-ray phase contrast imaging can be used for the $3 \mathrm{D}$ visualization and basic quantification of $\mathrm{A} \beta$ plaques in the mouse brain. At the same synchrotron facility the Pinzer et al. ${ }^{7}$ analyzed the neocortex of mouse brain via differential phase contrast imaging and accompanied their findings with complementary thioflavin $S$ staining of the brain to confirm the presence of the $\mathrm{A} \beta$ plaques in brain in CT data. The diffraction enhanced imaging phase contrast X-ray imaging technique was applied by the Connor et al. $^{8}$ at National Synchrotron Light Source, Brookhaven National Laboratory, USA. In that study, several scans of the one brain were needed in order to obtain a full scan of the brain. A comparison of the affected and wild type mouse brain was shown and the identity of $A \beta$ plaques was confirmed by applying specific immunostaining on the $\mathrm{A} \beta$ plaques. The $\mathrm{X}$-ray phase contrast tomography was applied for the purpose of the amyloid plaque imaging specifically to describe the microenvironment of the $A \beta$ plaque at European Synchrotron Radiation Facility, Grenoble, France ${ }^{9}$. Finally, the research group of Noda-Saita et al. ${ }^{10}$ at High Energy Accelerator Research Organization, Ibaraki, Japan used phase-contrast X-ray CT imaging to describe the density of $A \beta$ plaques supporting their findings with a complementary immunodetection of $A \beta$ plaques in the mouse brain and with scans of control brains.

Unfortunately, the synchrotron facilities are not readily available for general use since the complicated imaging setups imply higher costs of equipment, its service and its maintenance. Moreover, the multiple scans have to be acquired to retrieve the information from the whole brain sample because of the sample's size limitations. This restricts a wide use of synchrotron based CT imaging in the Alzheimer's disease research, despite the obvious advantages for the quantification of amyloid plaques. 


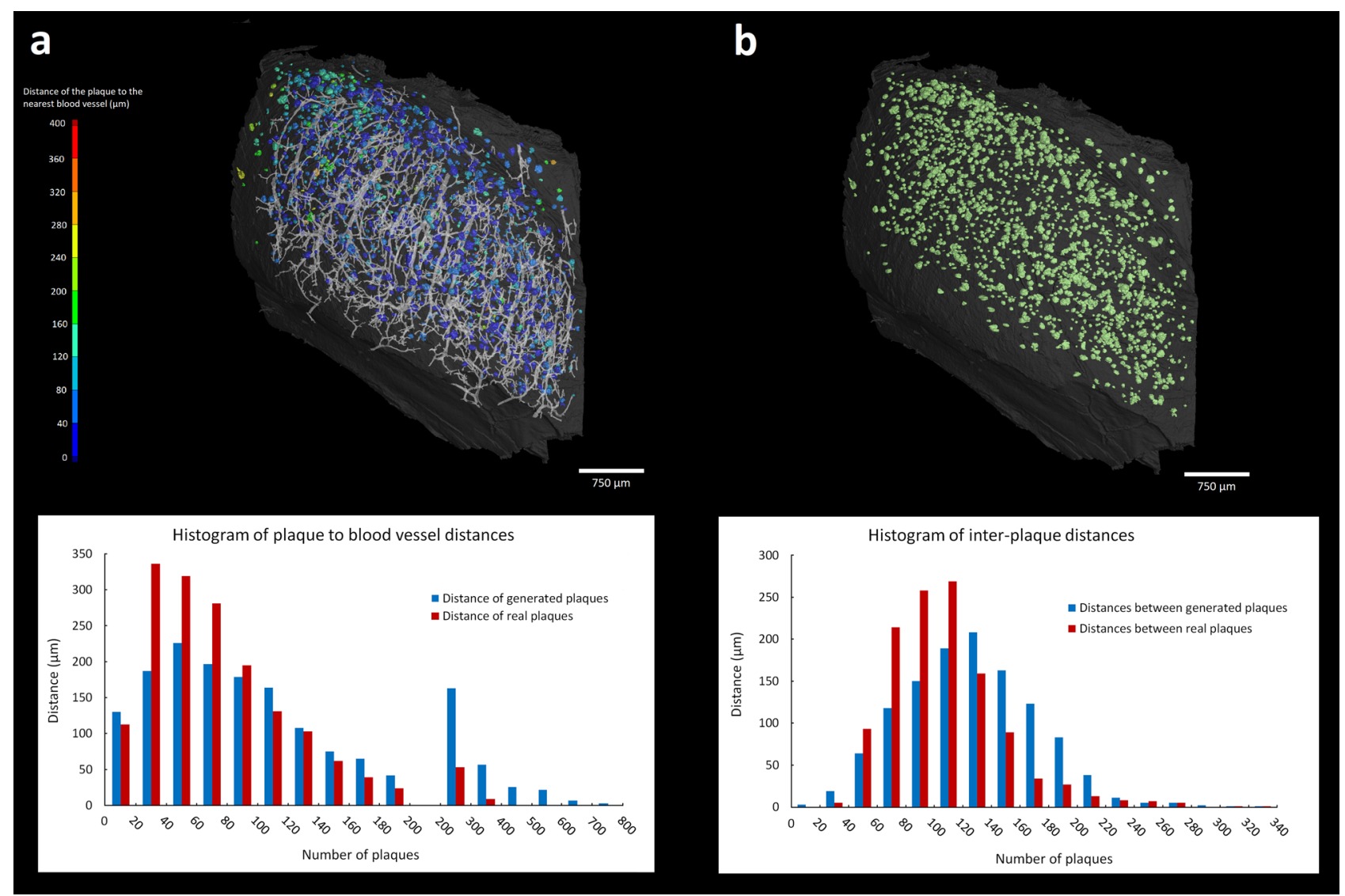

Figure 5. 3D quantitative analysis of the plaques distribution in hippocampus: Horizontal view of the hippocampus with color coded distances of individual plaques to the nearest blood vessel (a) and the corresponding histogram showing the distances to the nearest blood vessel of randomly generated (in blue) and the real (in red) plaques, respectively. Horizontal view of the hippocampus depicting the distribution of the plaques inside the hippocampus (b) and the corresponding histogram showing the distribution of randomly generated plaques (in blue), and the distribution of real plaques (in red) ${ }^{41,42}$.

This fact led us to the idea of developing an imaging method for detection of amyloid plaques in the rat brain applicable to easily accessible industrial X-ray lab-based devices. Absorption based contrast enhanced $\mathrm{X}$-ray micro CT imaging as an alternative to the synchrotron based phase contrast imaging techniques retrieves not only a 3D distribution of amyloid plaques with decent voxel resolution, but also provides valuable data for quantitative analysis of the amyloid plaques (total number, volume, shape).

The immunodetection of amyloid plaques performed on histological slices is the golden standard of identification of plaques in the brain tissue. In this paper, we used this method to validate our findings. In order to precisely correlate the micro CT data with the immunodetection of the plaques, we used the brain which had been previously scanned on micro CT. Then, we performed a classical histological sectioning and carried out the immunodetection of the plaques. The comparison of data from the same brain analyzed by both techniques showed a good agreement between immunodetection and micro CT imaging. Even though the immunodetection is indisputably more sensitive in detection of smaller plaques, it lacks a convenient approach to the 3D analysis across large brain volumes. This comparison thus highlights the advantages of contrast enhanced micro CT imaging. The immunostaining based affirmation of plaque identity detected by micro CT shows that the absorption-based contrast enhanced micro CT imaging is robust in detection of amyloid plaques. While its applicability as described in this report is not suitable for in vivo experiments for the staining protocol toxicity or because of the high irradiation dose, we believe the CT brain imaging approach in the context of Alzheimer's disease research has a considerable potential for further development.

\section{Materials and methods}

Animals. Brains from three 18 months old female rats (two transgenic TgF-344 AD animals and one wild type control) obtained from the local breeding colony at Faculty of Medicine in Pilsen, Charles University, were used to collect the data. All protocols followed in this study were approved by the Ethical Committee of the Ministry of Education, Youth and Sports of the Czech Republic (approval no. MSMT-12048/2019-14) according to the Guide for the Care and Use of Laboratory Animals (Protection of Animals from Cruelty Law Act No. 246/92, Czech Republic). 


\begin{tabular}{|l|l|l|l|l|}
\hline Sample & Rat brain F60+ & Rat brain F61- & Rat brain F87 + & Hippocampus F87 + \\
\hline Voltage $[\mathrm{kV}]$ & 60 & 60 & 60 & 60 \\
\hline Current $[\mu \mathrm{A}]$ & 200 & 200 & 200 & 200 \\
\hline Timing $[\mathrm{ms}]$ & 600 & 600 & 600 & 700 \\
\hline Source spot size $[\mu \mathrm{m}]$ & 5 & 5 & 5 & 5 \\
\hline Sample/source distance $[\mathrm{mm}]$ & 40.8 & 40.8 & 40.8 & 11.9 \\
\hline Sample/detector distance $[\mathrm{mm}]$ & 864.9 & 864.9 & 864.9 & 785.4 \\
\hline Images & 2200 & 2200 & 2200 & 2400 \\
\hline Time $[\mathrm{min}]$. & 80 & 80 & 80 & 100 \\
\hline Voxel size $\left[\mu \mathrm{m}^{3}\right]$ & 9 & 9 & 9 & 3 \\
\hline
\end{tabular}

Table 1. Micro CT scan settings.

Micro CT staining. At the beginning of the experiments the rats were overdosed with pentobarbital and intracardially perfused with saline, followed by $4 \%$ PFA. Then the brains were manually extracted and post-fixed in $4 \%$ PFA for another $3 \mathrm{~h}$. Afterwards, the samples were dehydrated in ethanol solutions of different concentrations which increased: $30 \%, 50 \%, 70 \%, 80 \%$ and $90 \%$. The process lasted $12 \mathrm{~h}$ for each concentration. After the dehydration, samples were submerged in a staining solution consisting of $1 \%$ iodine in $90 \%$ methanol for $72 \mathrm{~h}$, where the staining solution was refreshed after the first $24 \mathrm{~h}$. Then the samples were washed in $50 \%$ ethanol and embedded in $1 \%$ agarose gel. In one of the transgenic animals, after the whole brain scan, the dorsal hippocampus was dissected and embedded in $1 \%$ agarose gel for another scan.

Micro CT measurement. Before scanning, all samples were embedded in 1\% agarose gel in $15 \mathrm{ml}$ Falcon tubes in order to prevent movement during the imaging procedure. The micro CT scanning was performed using a laboratory system GE Phoenix v|tome|x L 240 (GE Sensing \& Inspection Technologies GmbH, Germany), equipped with a $180 \mathrm{kV} / 15 \mathrm{~W}$ maximum power nanofocus X-ray tube and a high contrast flat panel detector dynamic 41|100 (number of pixels: $4048 \times 4048 \mathrm{px}$, pixel size $100 \mu \mathrm{m}$ ). The measurements were carried out in an air-conditioned cabinet $\left(21^{\circ} \mathrm{C}\right)$. The parameters for each scan are indicated in the Table 1 . The tomographic reconstruction was realized by software GE phoenix datos $\mid \times 2.0$ (GE Sensing \& Inspection Technologies $\mathrm{GmbH})$.

Micro CT data processing. All 3D visualizations and measurements were performed in VG Studio MAX 3.4 software (Volume Graphics $\mathrm{GmbH})^{41}$. The segmentation of plaques in the hippocampus sample was carried out using Avizo 9.5 software (Thermo Fisher Scientific) ${ }^{42}$. In the first step we isolated the hippocampus from the background by creating the corresponding region of interest (ROI). Blood vessels were segmented by global thresholding based on the grey level (vessels appeared as the darkest part since they did not contain any iodine), continuity, and their resulting prolonged and branched 3D shape. The amyoid plaques were segmented manually in Avizo 9.5 software by selecting the plaque areas across the sections under the following criteria: the area of the plaque should be distinguishably darker then the surrounding tissue and its volume should extend across at least 3 following sections. The boundaries of smaller plaques were selected section by section, in bigger plaques (some were spread over more than 30 sections) we interpolated between every other slice while any inconsistencies were additionally manually corrected.

For the analysis of $\mathrm{A} \beta$ distribution we generated datasets of plaques coordinates (Matlab R2020a). First, the coordinates of plaques centroids were extracted from the segmented 3D binary data to represent the experimental dataset. Then the random dataset of "plaque's" centroids was created within the volume of the sample leaving out its vascular system. The resulting coordinates then represented centroids of randomly distributed A $\beta$ plaques with total amount corresponding to the experimental dataset. The analysis of $\mathrm{A} \beta$ plaque position in relation to the blood vessels was carried out in VG studio. Both real plaques centroid positions and simulated plaques centroid positions were imported in form of binary image data. The distance of the centroid to the nearest blood vessel was measured via pore analysis module. Subsequently, the intra-plaques distances were analyzed separately in simulated plaque centroids and real plaque centroids. The nearest neighboring centroid of each centroid was found using 3D Euclidean distances analysis in Matlab R2020a. Repeating combinations of the nearest neighbors were excluded from the subsequent analysis.

Statistical analysis. The non-parametric Mann-Whitney test was applied to compare the detected and random datasets (https://www.statskingdom.com/170median_mann_whitney.html).

Histology and immunohistochemical labeling. After the CT measurements, the sample tissue was embedded in a paraffin block and cut into $10 \mu$ thick sections using an Automated Microtome (Leica RM2255). The slices were then deparaffinized and rehydrated. Phosphate buffer saline (PBS, $0.1 \mathrm{M}$ ) was applied for washing. To block the non-specific binding, normal goat serum (ab138478, Abcam) was used. The sections were then incubated overnight at $4{ }^{\circ} \mathrm{C}$ with the primary antibody (ab2539, Abcam, 1:200, $1 \mathrm{mg} / \mathrm{ml}$ ) against amyloid beta. Next day, the sections were washed with 1 X PBS $(0.01 \mathrm{M})$ thrice $\left(5^{\prime}, 10^{\prime}\right.$ and $\left.15^{\prime}\right)$ to remove the unbound anti- 
body remnants. Staining was visualized with an Alexa Fluor 647-conjugated goat anti-rabbit antibody (Jackson ImmunoResearch Laboratories) applied in 1:500 dilution at $37^{\circ} \mathrm{C}$ for $4 \mathrm{~h}$. All the sections were then counterstained with DAPI to label the nuclei and glass mounted using fluoroshield mounting medium (Merck). The Amyloid beta plaques were visualized using fluorescent microscopy (Olympus), and quantified in open source Fiji image analysis software ${ }^{43}$.

\section{Data availability}

Datasets used in this publication are available on request at corresponding authors.

Received: 3 December 2020; Accepted: 18 February 2021

Published online: 16 March 2021

\section{References}

1. Hardy, J. \& Selkoe, D. J. The amyloid hypothesis of Alzheimer's disease: Progress and problems on the road to therapeutics. Science 297(5580), 353-356 (2002)

2. Kametani, F. \& Hasegawa, M. Reconsideration of amyloid hypothesis and tau hypothesis in Alzheimer's disease. Front. Neurosci. 12, 25 (2018).

3. Zempel, H. \& Mandelkow, E. Lost after translation: Missorting of Tau protein and consequences for Alzheimer disease. Trends Neurosci. 37(12), 721-732 (2014)

4. Hardy, J. A. \& Higgins, G. A. Alzheimer's disease: The amyloid cascade hypothesis. Science 256(5054), 184-186 (1992).

5. Cao, J., Hou, J., Ping, J. \& Cai, D. Advances in developing novel therapeutic strategies for Alzheimer's disease. Mol. Neurodegen. 13(1), 64 (2018).

6. Astolfo, A., Lathuiliere, A., Laversenne, V., Schneider, B. \& Stampanoni, M. Amyloid- $\beta$ plaque deposition measured using propagation-based X-ray phase contrast CT imaging. J. Synchr. Radiat. 23(3), 813-819 (2016).

7. Pinzer, B. R. et al. Imaging brain amyloid deposition using grating-based differential phase contrast tomography. Neuroimage. 61(4), 1336-1346 (2012).

8. Connor, D. M. et al. Computed tomography of amyloid plaques in a mouse model of Alzheimer's disease using diffraction enhanced imaging. Neuroimage. 46(4), 908-914 (2009).

9. Massimi, L. et al. Exploring Alzheimer's disease mouse brain through X-ray phase contrast tomography: From the cell to the organ. NeuroImage. 184, 490-495 (2019).

10. Noda-Saita, K. et al. Quantitative analysis of amyloid plaques in a mouse model of Alzheimer's disease by phase-contrast X-ray computed tomography. Neuroscience 138(4), 1205-1213 (2006).

11. Massimi, L. et al. Assessment of plaque morphology in Alzheimer's mouse cerebellum using three-dimensional X-ray phase-based virtual histology. Sci. Rep. 10(1), 1-10 (2020).

12. Metscher, B. D. MicroCT for developmental biology: A versatile tool for high-contrast 3D imaging at histological resolutions. Dev. Dyn. 238(3), 632-640 (2009).

13. Metscher, B. D. MicroCT for comparative morphology: Simple staining methods allow high-contrast 3D imaging of diverse nonmineralized animal tissues. BMC Physiol. 9(1), 11 (2009).

14. Li, L. et al. Superficial cells are self-renewing chondrocyte progenitors, which form the articular cartilage in juvenile mice. FASEB J. 31(3), 1067-1084 (2017).

15. Kaucka, M. et al. Oriented clonal cell dynamics enables accurate growth and shaping of vertebrate cartilage. Elife. 6, e25902 (2017).

16. Kaucka, M. et al. Analysis of neural crest-derived clones reveals novel aspects of facial development. Sci. Adv. 2(8), e1600060 (2016).

17. Kaucka, M. et al. Signals from the brain and olfactory epithelium control shaping of the mammalian nasal capsule cartilage. Elife. 7, e34465 (2018).

18. Celá, P. et al. Ciliopathy protein Tmem107 plays multiple roles in craniofacial development. J. Dent. Res. 97(1), 108-117 (2018).

19. Hampl, M. et al. Polarized sonic hedgehog protein localization and a shift in the expression of region-specific molecules is associated with the secondary palate development in the veiled chameleon. Front. Cell Dev. Biol. 8, 572 (2020).

20. Landova Sulcova, M. et al. Developmental mechanisms driving complex tooth shape in reptiles. Dev. Dyn. 249(4), 441-464 (2020).

21. Kohoutek, J. et al. Mouse model of congenital heart defects, dysmorphic facial features and intellectual developmental disorders as a result of nonfunctional CDK13. Front. Cell Dev. Biol. 7, 155 (2019).

22. Heude, E. et al. Unique morphogenetic signatures define mammalian neck muscles and associated connective tissues. Elife. 7 , e40179 (2018).

23. Vymazalová, K., Vargová, L., Zikmund, T. \& Kaiser, J. The possibilities of studying human embryos and foetuses using micro-CT: A technical note. Anat. Sci. Int. 92(2), 299-303 (2017).

24. Tesařová, M. et al. An interactive and intuitive visualisation method for X-ray computed tomography data of biological samples in 3D Portable Document Format. Sci. Rep. 9(1), 1-8 (2019).

25. Stolz, E. et al. Angioarchitectural changes in subacute cerebral venous thrombosis A synchrotron-based micro-and nano-CT study. Neuroimage 54(3), 1881-1886 (2011).

26. Heinzer, S. et al. Hierarchical microimaging for multiscale analysis of large vascular networks. Neuroimage. 32(2), 626-636 (2006).

27. Ghanavati, S., Lisa, X. Y., Lerch, J. P. \& Sled, J. G. A perfusion procedure for imaging of the mouse cerebral vasculature by X-ray micro-CT. J. Neurosci. Methods 221, 70-77 (2014).

28. Langheinrich, A. C. et al. Evaluation of the middle cerebral artery occlusion techniques in the rat by in-vitro 3-dimensional microand nano computed tomography. BMC Neurol. 10(1), 36 (2010).

29. Chugh, B. P. et al. Measurement of cerebral blood volume in mouse brain regions using micro-computed tomography. Neuroimage. 47(4), 1312-1318 (2009).

30. Xie, B., Miao, P., Sun, Y., Wang, Y. \& Yang, G. Y. Micro-computed tomography for hemorrhage disruption of mouse brain vasculature. Transl. Stroke Res. 3(1), 174-179 (2012).

31. Dorr, A., Sled, J. G. \& Kabani, N. Three-dimensional cerebral vasculature of the CBA mouse brain: A magnetic resonance imaging and micro computed tomography study. Neuroimage. 35(4), 1409-1423 (2007).

32. Hayasaka, N. et al. In vivo diagnostic imaging using micro-CT: sequential and comparative evaluation of rodent models for hepatic/ brain ischemia and stroke. PLoS ONE 7(2), e32342 (2012).

33. Dobrivojević, M., Bohaček, I., Erjavec, I., Gorup, D. \& Gajović, S. Computed microtomography visualization and quantification of mouse ischemic brain lesion by nonionic radio contrast agents. Croatian Med. J. 54(1), 3-11 (2013).

34. Anderson, R. \& Maga, A. M. A novel procedure for rapid imaging of adult mouse brains with microCT using iodine-based contrast. PLOS ONE 10(11), e0142974 (2015).

35. de Crespigny, A. et al. 3D micro-CT imaging of the postmortem brain. J. Neurosci. Methods 171(2), 207-213 (2008). 
36. Saito, S. \& Murase, K. Ex vivo imaging of mouse brain using micro-CT with non-ionic iodinated contrast agent: A comparison with myelin staining. Br. J. Radiol. 85(1019), e973-e978 (2012).

37. Bautista, N. S. et al. Ex vivo micro-CT imaging of murine brain models using non-ionic iodinated contrast. Am. Inst. Phys. Conf. Proc. 1(1626), 197-200 (2014).

38. Hainfeld, J. F. et al. Gold nanoparticle imaging and radiotherapy of brain tumors in mice. Nanomedicine. 8(10), 1601-1609 (2013).

39. Kastriti, M. E. et al. Ablation of CNTN2+ pyramidal neurons during development results in defects in neocortical size and axonal tract formation. Front. Cell. Neurosci. 13, 454 (2019).

40. Zikmund, T. et al. High-contrast differentiation resolution 3D imaging of rodent brain by X-ray computed microtomography. J. Instrum. 13(02), C02039 (2018).

41. Volume Graphics. https://www.volumegraphics.com 2020 (accessed 30 October 2020).

42. Thermo Fisher Scientific. https://www.fei.com/software/avizo3d/.

43. Fiji. https://imagej.net/Fiji.

\section{Acknowledgements}

This research was carried out under the project CEITEC 2020 (LQ1601) with financial support from the Ministry of Education, Youth and Sports of the Czech Republic under the National Sustainability Programme II and CzechNanoLab Research Infrastructure supported by MEYS CR (LM2018110), projects PRIMUS 27051, Progres Q39, FIND No. CZ.02.1.01/0.0/0.0/16_019/0000787 provided by the Ministry of Education, Youth and Sports of the Czech Republic, and GAUK 12539. We are grateful to Marketa Slajerova for help with processing the samples.

\section{Author contributions}

M.K., T.Z., J.K. and K.J. designed the study, M.K. performed the CT scanning and staining, M.K. and J.S. analyzed the CT data, A.K. and S.L.P.P. processed and analyzed the histology, M.K. and K.J. wrote the manuscript.

\section{Competing interests}

The authors declare no competing interests.

\section{Additional information}

Supplementary Information The online version contains supplementary material available at https://doi. org/10.1038/s41598-021-84579-x.

Correspondence and requests for materials should be addressed to M.K. or K.J.

Reprints and permissions information is available at www.nature.com/reprints.

Publisher's note Springer Nature remains neutral with regard to jurisdictional claims in published maps and institutional affiliations.

(c) (i) Open Access This article is licensed under a Creative Commons Attribution 4.0 International License, which permits use, sharing, adaptation, distribution and reproduction in any medium or format, as long as you give appropriate credit to the original author(s) and the source, provide a link to the Creative Commons licence, and indicate if changes were made. The images or other third party material in this article are included in the article's Creative Commons licence, unless indicated otherwise in a credit line to the material. If material is not included in the article's Creative Commons licence and your intended use is not permitted by statutory regulation or exceeds the permitted use, you will need to obtain permission directly from the copyright holder. To view a copy of this licence, visit http://creativecommons.org/licenses/by/4.0/.

(C) The Author(s) 2021 